\title{
MULTI-PEAK SOLUTIONS TO TWO TYPES OF FREE BOUNDARY PROBLEMS
}

\author{
YI LI AND SHUANGJIE PENG
}

\begin{abstract}
We consider the existence of multi-peak solutions to two types of free boundary problems arising in confined plasma and steady vortex pair under conditions on the nonlinearity we believe to be almost optimal. Our results show that the "core" of the solution has multiple connected components, whose boundary called free boundary of the problems consists approximately of spheres which shrink to distinct single points as the parameter tends to zero.
\end{abstract}

\section{INTRODUCTION}

In this paper, we consider the following partial differential equation with Dirichlet boundary condition

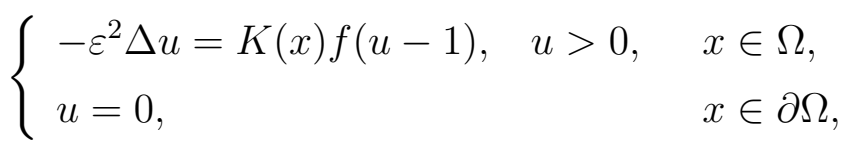

where $\Omega \subset \mathbb{R}^{N}(N \geq 3)$ is a bounded domain with smooth boundary $\partial \Omega, \varepsilon \in \mathbb{R}_{+}$is a small parameter, $f(t)$ is continuous in $t$ and $f(t)=0$ for $t \leq 0, K(x)$ is a positive function in $\bar{\Omega}$. For a positive solution $u_{\varepsilon}$ to (1.1), the boundary of the core $A_{\varepsilon}:=\left\{u_{\varepsilon}>1\right\}$ is the free boundary in this problem.

Problem (1.1) is related to the following simple model describing the equilibrium of a plasma confined in a toroidal cavity (a "Tokomak machine"):

$$
\begin{array}{ll}
\Delta v=\lambda g(x, v) & x \in \Omega, \\
v=c & x \in \partial \Omega, \\
\int_{\partial \Omega} \frac{\partial v}{\partial \nu} d s=I, &
\end{array}
$$

where $\nu$ is the outward unit normal to $\partial \Omega, g(x, t)=0$ for $t \geq 0, c$ is a constant which is unprescribed, and $I$ is a given positive constant. For a detailed presentation of this model, the reader is referred to the Appendix in [33].

In (1.2), we can suppose that $c>0$, since this is necessary for the existence of solutions to problem (1.2) for large $\lambda$ (see [34]). Let $v=c(1-u)$, we find that if $v$ satisfies problem (1.2), then $u$ solves

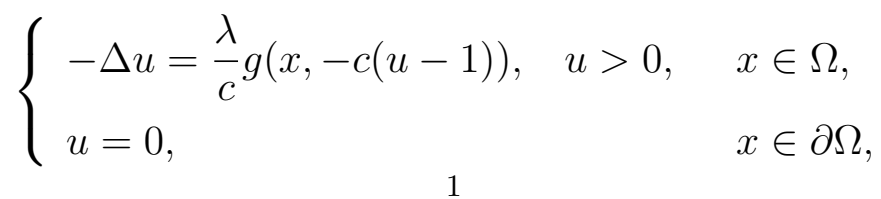


and $c$ satisfies

$$
I=\int_{\partial \Omega} \frac{\partial v}{\partial \nu} d s=-c \int_{\partial \Omega} \frac{\partial u}{\partial \nu} d s=\lambda \int_{\Omega} g(x,-c(u-1)),
$$

and $I>0$ if $g(x, t)>0$ for $t<0$. Hence, we see problem (1.1) is of the form of problem (1.3) with $\varepsilon^{2}=c / \lambda$.

Problem (1.2) was studied by many authors. One example is $g(x, v)=K(x) v_{-}$, where $v_{+}=\max \{v, 0\}, v=v_{+}-v_{-}$. In [33, 34], the solutions were obtained by minimizing a certain variational problem. When $N=2$ and $N \geq 3$, and $K(x) \equiv 1$, for solutions obtained in [33, 34], Caffarelli and Friedman [8] and Shibata [31] investigated precise asymptotic location and shape of the free boundary as $\lambda \rightarrow+\infty$. Especially, they proved that for $\lambda$ sufficiently large, the core is approximated by a ball with the center converging to a harmonic center and the radius being comparable to $1 / \sqrt{\lambda}$. In [12], Flucher and Wei considered problem (1.1) for the case $K(x) \equiv 1, N \geq 3$, and $f(t)=t_{+}^{p}(1<p<(N+$ $2) /(N-2))$, and proved that for $\varepsilon$ sufficient small, a mountain pass solution and its core have the similar asymptotic behaviors as those in [8]. Problem (1.1) was also studied by Shibata in the case $N \geq 3$ and $f(t)=t_{+}^{p}(1 \leq p<(N+2) /(N-2))$ in [32], where Shibata proved that the least energy solution concentrates at a global maximum of $K(x)$ as $\varepsilon \rightarrow 0$. We should also mention the interesting work [36] where Wei obtained multi-peak solutions to (1.1) by gluing localized solutions. More results can be found in $[4,22,23]$ and the references therein.

However, for the general nonlinearity $f$, it seems that there are very few results. Moreover, even for some special nonlinearity $f(t)$ ( for example, $f(t)=t_{+}$, or $t_{+}^{p}$ ), the core of the solutions is simply connected. Recently, for the case $K(x) \equiv 1, f(t)=t_{+}$and $N=2$, under the condition that the homology of $\Omega$ is nontrivial, Cao, Peng and Yan [9] proved by a constructive way that for any given integer $k \geq 1$, there is $\varepsilon_{0}>0$, such that for $0<\varepsilon<\varepsilon_{0},(1.1)$ has a solution with core consisting of $k$ components. It follows from [33], that the solution for problem (1.1) is unique in the case that $f(t)=t_{+}$and $\varepsilon$ is large, so it is a natural problem whether or not problem (1.1) has multiple solutions for small $\varepsilon$. The main purpose of this paper is trying to find multiple solutions for much more general nonlinearities. More precisely, under some conditions on $f(t)$, which we believe to be almost optimal, we prove that problem (1.1) has solutions which concentrate on distinct points in $\bar{\Omega}$ as $\varepsilon \rightarrow 0$, which implies that the core of the solutions has several connected components.

We suppose that $K(x)$ has $k$ sets of local maxima in $\bar{\Omega}$ including on the boundary $\partial \Omega$, that is,

$\left(K_{1}\right)$ there are $M^{i} \subset \bar{\Omega}(i=1, \cdots, k)$ satisfying

$$
K(x)=m_{i}, \forall x \in M^{i}
$$

$\left(K_{2}\right)$ there are bounded disjoint open sets $O^{i} \subset \Omega(i=1, \cdots, k)$ satisfying

$$
M^{i} \subset \bar{O}^{i}, m_{i}=\sup _{x \in O^{i}} K(x)>\sup _{\partial O^{i} \backslash \partial \Omega} K(x) .
$$

We also assume that $f: \mathbb{R} \rightarrow \mathbb{R}$ is Lipschtiz continuous and satisfies

$\left(f_{1}\right) \quad f(t)=0$ for $t \leq 0$ and $\limsup _{t \rightarrow+\infty} \frac{f(t)}{t^{l}} \leq 0$, where $l=(N+2) /(N-2)$. 
$\left(f_{2}\right)$ There exists $\xi \geq 0$ such that $F(\xi)>0$, where $F(t)=\int_{0}^{t} f(s) d s$. Let $\xi_{0}=\inf \{\xi \geq$ $0: F(\xi)>0\}$.

We define $H_{\varepsilon}$ to be the complete of $C_{0}^{\infty}(\Omega)$ with respect to the norm $\|u\|_{\varepsilon}=\left(\int_{\Omega} \varepsilon^{2}|\nabla u|^{2}\right)^{1 / 2}$, and $D^{1,2}\left(\mathbb{R}^{N}\right), D_{\varepsilon}^{1,2}\left(\mathbb{R}^{N}\right)$ the completes of $C_{0}^{\infty}\left(\mathbb{R}^{N}\right)$ with respect to the norms $\|u\|_{D^{1,2}\left(\mathbb{R}^{N}\right)}=$ $\left(\int_{\mathbb{R}^{N}}|\nabla u|^{2}\right)^{1 / 2}$ and $\|u\|_{D_{\varepsilon}^{1,2}\left(\mathbb{R}^{N}\right)}=\left(\int_{\mathbb{R}^{N}} \varepsilon^{2}|\nabla u|^{2}\right)^{1 / 2}$ respectively.

Theorem 1.1. Suppose that $\left(K_{1}\right)-\left(K_{2}\right)$ and $\left(f_{1}\right)-\left(f_{2}\right)$ hold. Then there exists $\varepsilon_{0}>0$, such that for $\varepsilon \in\left(0, \varepsilon_{0}\right)$, problem (1.1) has a positive solution $u_{\varepsilon}$ satisfying

(i) $\quad u_{\varepsilon}$ has $k$ local maximum points $x_{\varepsilon}^{i} \in O^{i}$ such that

$$
\lim _{\varepsilon \rightarrow 0} \max _{i=1, \cdots, k} \operatorname{dist}\left(x_{\varepsilon}^{i}, M^{i}\right)=0 \text {, }
$$

and

$$
u_{\varepsilon}(x) \leq C \varepsilon^{N-2} \max _{i=1, \cdots, k} \frac{1}{\left|x-x_{\varepsilon}^{i}\right|^{N-2}}, \forall x \in \Omega \backslash \bigcup_{i=1}^{k} B_{\delta}\left(x_{\varepsilon}^{i}\right),
$$

where $\delta>0$ is any fixed constant and $C$ depends on $\delta$.

(ii) for any sequence $\left\{\varepsilon_{n}\right\} \subset\left(0, \varepsilon_{0}\right)$ with $\varepsilon_{n} \rightarrow 0$, there exists a subsequence still denoted by $\left\{\varepsilon_{n}\right\}$, such that for each $i \in\{1, \cdots, k\}$, there is $x^{i} \in M^{i}$ with $x_{\varepsilon_{n}}^{i} \rightarrow x^{i}$ and $U^{i} \in$ $D^{1,2}\left(\mathbb{R}^{N}\right)$, a least energy solution of

$$
-\Delta U=m_{i} f(U-1), \quad U>0, U \in D^{1,2}\left(\mathbb{R}^{N}\right),
$$

satisfying

$$
u_{\varepsilon_{n}}=\sum_{i=1}^{k} U^{i}\left(\frac{x-x_{\varepsilon_{n}}^{i}}{\varepsilon_{n}}\right)+\omega_{\varepsilon_{n}},
$$

where $\omega_{\varepsilon_{n}} \in D_{\varepsilon_{n}}^{1,2}\left(\mathbb{R}^{N}\right)$, and $\left\|\omega_{\varepsilon_{n}}\right\|_{D_{\varepsilon_{n}}^{1,2}\left(\mathbb{R}^{N}\right)}=o\left(\varepsilon_{n}^{N / 2}\right)$.

In this paper, we also consider the following problem

$$
\begin{cases}-\varepsilon^{2} \Delta u=f(u-k(x)), & x \in \Omega, \\ u=0, & x \in \partial \Omega .\end{cases}
$$

Problem (1.6) is a variant of the following free-boundary problem arising in steady vortex pairs:

$$
\begin{aligned}
& -\Delta \Psi= \begin{cases}\lambda f(\Psi), & x \in A, \\
0, & x \in \Omega \backslash \bar{A},\end{cases} \\
& \left.\Psi\right|_{\partial A}=0,\left.\quad \Psi\right|_{\partial \Omega}=-k_{0}(x)<0,
\end{aligned}
$$

where $(\Psi, A)$ is a steady vortex pair: $\Psi$ is a Stokes stream function, $A \subset \Omega \subset \mathbb{R}^{N}$ is an open set called the cross-section of a steady vortex ring and unknown a priori. $k_{0}(x)>0$ is a continuous function defined on $\partial \Omega$. For more detailed presentation of this model, we refer the readers to [17] and [28]. 
Let $\varphi$ be the solution of

$$
\begin{cases}\Delta k(x)=0, & x \in \Omega, \\ k(x)=k_{0}(x), & x \in \partial \Omega .\end{cases}
$$

Then, $k(x)>0$ achieves its maximum and minimum on $\Omega$.

Let $u=\Psi+k(x), A=\{x \in \Omega: u>k(x)\}$ and $\varepsilon^{2}=1 / \lambda$, then problems (1.7) and (1.8) become (1.6).

In this paper, we investigate problem (1.6) to obtain its solution pairs $\left(u_{\varepsilon}, A_{\varepsilon}\right)$ for $\varepsilon$ sufficiently small, where the "vortex core" $A_{\varepsilon}:=\left\{x \in \Omega: u_{\varepsilon}>k(x)\right\}$.

There are many existence results for problem (1.6) under various assumptions. In $[1,2$, $27,38]$, the solutions were obtained by using mountain pass lemma for various nonlinearities $f(x, u)$ and any $\varepsilon>0$. In $[3,6,17,28,35]$, to find the solutions, the constrained variation methods were used, but the vorticity function $f$ is unknown a priori. Moreover, in $[6$, $17,28]$, the solutions were obtained by regarding $1 / \varepsilon^{2}$ as eigenvalue, so $\varepsilon$ is not arbitrary. The asymptotic behavior of the solution pair $\left(u_{\varepsilon}, A_{\varepsilon}\right)$ of problem (1.6) was investigated in $[6,8,12,24,38]$. Recently, for the case $f(t)=t_{+}^{p-1}$ and $N \geq 2$, under the condition that $k(x)$ has $l$ strictly local minimum points on the boundary $\partial \Omega$, the authors [25] proved that for $\varepsilon$ sufficiently small, (1.6) has a solution with "vortex core" consisting of $l$ components by a constructive way.

We also emphasize that almost all of the above papers imposed the Ambrosetti-Rabinowitz condition on the nonlinearity $f(t)$ and most of the solutions mentioned here are in some sense the least energy solutions and the "vortex core" shrinks to a single point as $\varepsilon \rightarrow 0$. In this paper, using the arguments in proving Theorem 1.1, we can find some high energy solutions whose "vortex core" consists of multiple connected components which shrink to distinct points in $\bar{\Omega}$ as $\varepsilon \rightarrow 0$ under the much more general conditions $\left(f_{1}\right)-\left(f_{2}\right)$.

Our following assumptions on $k(x)$ cover the case that $k(x) \in C^{1}(\bar{\Omega})$ is harmonic:

$\left(k_{1}\right)$ there are $M^{i} \subset \bar{\Omega}(i=1, \cdots, k)$ satisfying

$$
k(x)=a_{i}>0, \forall x \in M^{i}
$$

$\left(k_{2}\right)$ there are bounded disjoint open sets $O^{i} \subset \Omega(i=1, \cdots, k)$ satisfying

$$
M^{i} \subset \bar{O}^{i}, a_{i}=\inf _{x \in O^{i}} k(x)<\inf _{\partial O^{i} \backslash \partial \Omega} k(x) .
$$

Theorem 1.2. Suppose that $k(x)>0$ in $\bar{\Omega},\left(k_{1}\right)-\left(k_{2}\right)$ and $\left(f_{1}\right)-\left(f_{2}\right)$ hold and $f(t)$ is strictly increasing in positive $t$. Then Theorem 1.1 is true to problem (1.6) with $U^{i} \in$ $D^{1,2}\left(\mathbb{R}^{N}\right)$ being a least energy solution of

$$
-\Delta U=f\left(U-a_{i}\right), \quad U>0, U \in D^{1,2}\left(\mathbb{R}^{N}\right) .
$$

By (5.1)-(5.3) in [5], we see (1.4) or (1.10) has a lease energy solution if $\left(f_{1}\right)-\left(f_{2}\right)$ are satisfied, and also, similarly to [5], we can use Pohozaev's identity to check that $\left(f_{1}\right)-\left(f_{2}\right)$ are almost necessary for existence of a non-trivial solution of the associated problem (1.4). We point out here that in our theorems, if $\cup_{i}^{k} M^{i} \subset \Omega$, we do not require the Lipschitz continuity of $f(t)$ since in this case we do not need to use the blow-up technique near the boundary in Proposition 3.3. Furthermore, if we assume the uniqueness of the least energy 
solution of (1.4) or (1.10), it is not necessary to pass to subsequences in our theorems. At last, from the proof of Theorem 1.1, we see that Theorem 1.1 is still true for a unbounded domain $\Omega$.

By gluing localized solutions, we will search for a solution to (1.1) or (1.6) whose core consists of $k$ disjoint connected components close to the corresponding $M^{i}$, and the solution can be scaled into a least energy solution of (1.1) or (1.6) associated to the corresponding $M^{i}$. Thus, on one side, we need to use the least energy solutions of (1.1) or (1.6) to construct an approximate solution, which is an analogy of the Lyapounov-Schmidt reduction approach (see, for example, [13] ). On the other side, we apply the descent gradient flow in variational method to search for the critical point of the corresponding functional, which does not require any uniqueness result of the least energy solution nor isolatedness result of the least energy. We should point out that for gluing localized solutions, there have been many efforts starting from the pioneer works [10, 11, 15, 20, 30], for example, we can refer to [7, 29] and the references therein.

We remark that differently from [7], we should overcome some additional difficulties. Firstly, we need to know the properties of the least energy solutions to the limit equations, including the mountain pass characterization of the least energy solutions and the property of decay of the least energy solutions at infinity. Secondly, since the least energy solutions of the limit equations decay algebraically, we can not modify the functional corresponding to (1.1) as in [7]. Instead, considering the fact that $f(u-c)=0$ for $u \leq c$, we modify the original equation by multiplying the nonlinearity $f(t)$ by a characteristic function on a suitable set, which can force the concentration phenomena not to occur outside $O^{i}(i=$ $1, \cdots, k)$.

The paper is organized as follows: in Section 2, we will prove some properties of the least energy solutions to the limit problem related to problem (1.1) or (1.6). The proofs for the main results will be provided in Section 3.

\section{THE LIMIT PROBLEM}

In this section, we consider the limit problem

$$
-\Delta u=a f(u-c), u>0, u \in D^{1,2}\left(\mathbb{R}^{N}\right)
$$

where $a, c$ are two positive constants, $f(t): \mathbb{R} \rightarrow \mathbb{R}$ satisfies $\left(f_{1}\right)-\left(f_{2}\right)$.

In order to make $\int_{\mathbb{R}^{N}} F(u-c)$ of class $C^{1}$ and a meaningful functional for $D^{1,2}\left(\mathbb{R}^{N}\right)$, we modify $f(t)$ as in [5], that is, we modify $f(t-c)$ by letting $\tilde{f}(t-c)=f\left(t \wedge\left(t_{0}+c\right)-c\right)$ if there exists $t_{0}>\xi_{0}$ such that $f\left(t_{0}\right) \leq 0$. For simplicity, we again denote by $f(t)$ the modified function.

We recall that a solution $w(x)$ of $(2.1)$ is said to be a least energy solution, if

$$
I_{a, c}(w)=m_{a, c}:=\inf \left\{I_{a, c}(u): u \in D^{1,2}\left(\mathbb{R}^{N}\right) \backslash\{0\} \text { is a solution of }(2.1)\right\} .
$$

Here $I_{a, c}: D^{1,2}\left(\mathbb{R}^{N}\right) \rightarrow \mathbb{R}$ is the functional corresponding to $(2.1)$

$$
I_{a, c}(u)=\frac{1}{2} \int_{\mathbb{R}^{N}}|\nabla u|^{2}-\int_{\mathbb{R}^{N}} a F(u-c) .
$$


In the fundamental paper [5], by considering the minimization problem

$$
\min \left\{\int_{\mathbb{R}^{N}}|\nabla u|^{2}: \int_{\mathbb{R}^{N}} a F(u-c)=1\right\},
$$

Berestycki and Lions proved that under conditions $\left(f_{1}\right)-\left(f_{2}\right),(2.1)$ has a least energy solution $w(x) \in D^{1,2}\left(\mathbb{R}^{N}\right)$ satisfying $w(x)>0$. Moreover, for any solution $u(x) \in$ $D^{1,2}\left(\mathbb{R}^{N}\right)$ of $(2.1)$, the following Pohozaev identity holds:

$$
\frac{N-2}{2} \int_{\mathbb{R}^{N}}|\nabla u|^{2}=N \int_{\mathbb{R}^{N}} a F(u-c) .
$$

Firstly, we have

Lemma 2.1. $I_{a, c}(u)$ has a mountain pass geometry, that is,

$$
I_{a, c}(0)=0 .
$$

There exist $\rho_{0}>0$ and $\delta_{0}>0$ such that $I_{a, c}(u) \geq \delta_{0}$ for all $\|u\|_{D^{1,2\left(\mathbb{R}^{N}\right)}}=\rho_{0}$.

There exists $u_{0}>0$ such that $\left\|u_{0}\right\|_{D^{1,2}\left(\mathbb{R}^{N}\right)}>\rho_{0}$ and $I_{a, c}\left(u_{0}\right)<0$.

Proof. (2.4) is trivial.

By assumptions $\left(f_{1}\right)-\left(f_{2}\right)$, there exists $C>0$ such that

$$
f(s-c) \leq C|s|^{\frac{N+2}{N-2}} \text { for all } s \in \mathbb{R} .
$$

Thus, we have

$$
F(s-c) \leq C|s|^{\frac{2 N}{N-2}} \text { for all } s \in \mathbb{R} .
$$

It follows from the embedding $D^{1,2}\left(\mathbb{R}^{N}\right) \hookrightarrow L^{\frac{2 N}{N-2}}\left(\mathbb{R}^{N}\right)$,

$$
\begin{aligned}
I_{a, c}(u) & \geq \frac{1}{2} \int_{\mathbb{R}^{N}}|\nabla u|^{2}-C \int_{\mathbb{R}^{N}}|u|^{\frac{2 N}{N-2}} \\
& \geq \frac{1}{2}\|u\|_{D^{1,2}\left(\mathbb{R}^{N}\right)}^{2}-C^{\prime}\|u\|_{D^{1,2}\left(\mathbb{R}^{N}\right)}^{\frac{\not N}{N-2}}, \forall u \in D^{1,2}\left(\mathbb{R}^{N}\right) .
\end{aligned}
$$

Therefore choosing $\rho_{0}>0$ small, we find that (2.5) holds.

Let $w$ be a given least energy solution of (2.1). Define

$$
u_{t}(x)=w\left(\frac{x}{t}\right) \quad(t>0)
$$

we see

$$
I_{a, c}\left(u_{t}\right)=\frac{t^{N-2}}{2} \int_{\mathbb{R}^{N}}|\nabla w|^{2}-t^{N} \int_{\mathbb{R}^{N}} a F(w-c) .
$$

By the Pohozaev identity (2.3), we deduce that for sufficiently large $L>1, I_{a, c}\left(u_{L}(x)\right)<0$. Hence $u_{L}(x)$ satisfies $(2.6)$. 
From Lemma 2.1, we can define

$$
b_{a, c}=\inf _{\gamma \in \Gamma} \max _{t \in[0,1]} I_{a, c}(\gamma(t)),
$$

where

$$
\Gamma=\left\{\gamma(t) \in C\left([0,1], D^{1,2}\left(\mathbb{R}^{N}\right)\right): \gamma(0)=0, I_{a, c}(\gamma(1))<0\right\},
$$

we have $b_{a, c}>0$.

In the following, we will prove $b_{a, c}=m_{a, c}$ by using the arguments in [21].

Lemma 2.2. There exists a path $\gamma \in \Gamma$ satisfying

$$
w \in \gamma([0,1]) \text { and } \max _{t \in[0,1]} I(\gamma(t))=m_{a, c},
$$

where $w(x)$ is a least energy solution of (2.1).

Proof. By Proposition 2.5, $|w(x)| \leq C /|x|^{N-2}$ for $|x|$ large. Hence, we define

$$
\gamma(t)(x)= \begin{cases}w\left(\frac{x}{t}\right) & t>0 \\ 0 & t=0\end{cases}
$$

It is easy to check that

$$
\frac{d}{d t} I_{a, c}(\gamma(t))>0 \text { for } t \in(0,1) \text { and } \frac{d}{d t} I_{a, c}(\gamma(t))<0 \text { for } t>1 .
$$

Thus we can find a curve $\gamma(t):[0, L] \rightarrow D^{1,2}\left(\mathbb{R}^{N}\right)$ such that

$$
\gamma(0)=0, I_{a, c}(\gamma(L))<0, w \in \gamma([0, L]), \max _{t \in[0, L]} I_{a, c}(\gamma(t))=m_{a, c} .
$$

After a suitable scale change in $t$, we get the desired path $\gamma \in \Gamma$.

Define

$$
\mathcal{P}=\left\{u \in D^{1,2}\left(\mathbb{R}^{N}\right) \backslash\{0\}: \frac{N-2}{2} \int_{\mathbb{R}^{N}}|\nabla u|^{2}-N \int_{\mathbb{R}^{N}} a F(u-c)=0\right\},
$$

then, we have

\section{Lemma 2.3.}

$$
m_{a, c}=\inf _{u \in \mathcal{P}} I_{a, c}(u), \gamma([0,1]) \cap \mathcal{P} \neq \emptyset \text { for all } \gamma \in \Gamma
$$

Proof. Define

$$
\mathcal{S}=\left\{u \in D^{1,2}\left(\mathbb{R}^{N}\right): \int_{\mathbb{R}^{N}} a F(u-c)=1\right\}
$$

Set

$$
\Phi(u)=u\left(\frac{x}{t_{u}}\right), t_{u}=\sqrt{\frac{N-2}{2 N}}\|u\|_{D^{1,2}\left(\mathbb{R}^{N}\right)} .
$$

then $\Phi: \mathcal{S} \rightarrow \mathcal{P}$ is one-to-one, and

$$
I_{a, c}(\Phi(u))=\frac{1}{2} t_{u}^{N-2}\|u\|_{D^{1,2}\left(\mathbb{R}^{N}\right)}^{2}-t_{u}^{N} \int_{\mathbb{R}^{N}} a F(u-c)
$$




$$
=\frac{1}{N}\left(\frac{N-2}{2 N}\right)^{\frac{N-2}{2}}\|u\|_{D^{1,2}\left(\mathbb{R}^{N}\right)}^{N}, \forall u \in \mathcal{S} .
$$

Thus

$$
\inf _{u \in \mathcal{P}} I_{a, c}(u)=\inf _{u \in \mathcal{S}} I_{a, c}(\Phi(u))=\inf _{u \in \mathcal{S}} \frac{1}{N}\left(\frac{N-2}{2 N}\right)^{\frac{N-2}{2}}\|u\|_{D^{1,2}\left(\mathbb{R}^{N}\right)}^{N} .
$$

Now, recalling the proof of Theorem 4 in [5], we know $\inf _{u \in \mathcal{S}}\|u\|_{D^{1,2\left(\mathbb{R}^{N}\right)}}$ is achieved and $\Phi(u)$ is a corresponding least energy solution. Thus, $m_{a, c}=\inf _{u \in \mathcal{P}} I_{a, c}(u)$.

For any $\gamma(t) \in \Gamma$, we see

$$
\begin{aligned}
& \frac{N-2}{2}\|\gamma(0)\|_{D^{1,2}\left(\mathbb{R}^{N}\right)}^{2}-N \int_{\mathbb{R}^{N}} a F(\gamma(0)-c)=0, \\
& \frac{N-2}{2}\|\gamma(1)\|_{D^{1,2}\left(\mathbb{R}^{N}\right)}^{2}-N \int_{\mathbb{R}^{N}} a F(\gamma(1)-c)<0 .
\end{aligned}
$$

By (2.5), there exists $t_{0} \in[0,1]$ such that

$$
\left\|\gamma\left(t_{0}\right)\right\|_{D^{1,2}\left(\mathbb{R}^{N}\right)}>\rho_{0}, \frac{N-2}{2}\left\|\gamma\left(t_{0}\right)\right\|_{D^{1,2}\left(\mathbb{R}^{N}\right)}^{2}-N \int_{\mathbb{R}^{N}} a F\left(\gamma\left(t_{0}\right)-c\right)=0 .
$$

So, $\gamma([0,1]) \cap \mathcal{P} \neq \emptyset$.

Now combining Lemma 2.2 and Lemma 2.3, we can easily obtain

Proposition 2.4. $b_{a, c}=m_{a, c}, m_{a_{1}, c}<m_{a_{2}, c}$ if $a_{1}>a_{2}$; Moreover, if $f(t)$ is strictly increasing in positive $t$, then $m_{a, c_{1}}<m_{a, c_{2}}$ for $c_{1}<c_{2}$.

Proposition 2.5. Let $\mathcal{S}_{a, c}$ be the set of least energy solutions $U$ of (2.1) satisfying $U(0)=$ $\max _{x \in \mathbb{R}^{N}} U(x)$. Then $\mathcal{S}_{a, c}$ is compact in $D^{1,2}\left(\mathbb{R}^{N}\right)$. Moreover, there exists $C>0$ independent of $U \in \mathcal{S}_{a, c}$, such that

$$
U(x) \leq \frac{C}{|x|^{N-2}} \text { for }|x| \text { large enough }
$$

Proof. From Pohozaev's identity, we see $\left\{\|U\|_{D^{1,2}\left(\mathbb{R}^{N}\right)}: U \in \mathcal{S}_{a, c}\right\}$ is bounded. Then, we see from elliptic estimate (see [19]) that $\mathcal{S}_{a, c}$ is bound in $L^{\infty}\left(\mathbb{R}^{N}\right)$. Moreover, the maximum principle implies that $\mathcal{S}_{a, c}$ is bounded away from 0 in $L^{\infty}\left(\mathbb{R}^{N}\right)$ and $\|U\|_{L^{\infty}}>C$ for all $U \in \mathcal{S}_{a, c}$.

Now we claim that $\lim _{|x| \rightarrow \infty} U(x)=0$ uniformly for $U \in \mathcal{S}_{a, c}$. To the contrary, we assume that for some $\left\{U_{k}\right\}_{k=1}^{\infty} \subset \mathcal{S}_{a, c}$ and $\left\{x_{k}\right\}_{k=1}^{\infty} \subset \mathbb{R}^{N}$ with $\lim _{k \rightarrow \infty}\left|x_{k}\right|=\infty$, it holds $\liminf _{k \rightarrow \infty} U_{k}\left(x_{k}\right)>0$.

Define $V_{k}(x)=U_{k}\left(x+x_{k}\right)$. We see also from regularity theory of elliptic equations that for some $\beta>0,\left\{U_{k}, V_{k}\right\}_{k=1}^{\infty}$ is bounded in $C^{1, \beta}\left(\mathbb{R}^{N}\right)$. Then, taking a subsequence if necessary, we can assume that for some $U, V \in D^{1,2}\left(\mathbb{R}^{N}\right), U_{k}$ and $V_{k}$ converge respectively to $U$ and $V$ in $C_{\text {loc }}^{1}\left(\mathbb{R}^{N}\right)$ and weakly in $D^{1,2}\left(\mathbb{R}^{N}\right)$. Moreover $\max _{x \in \mathbb{R}^{N}} U \geq c$ and $\max _{x \in \mathbb{R}^{N}} V \geq c$. Indeed, if $\max _{x \in \mathbb{R}^{N}} U<c$ or $\max _{x \in \mathbb{R}^{N}} V<c$, then by Pohozaev 
identity, $\left\|U_{k}\right\|_{D^{1,2}\left(\mathbb{R}^{N}\right)}=\left\|V_{k}\right\|_{D^{1,2}\left(\mathbb{R}^{N}\right)}=0$ and $U_{k}=V_{k}=0$ which is a contradiction to $\liminf _{k \rightarrow \infty} U_{k}\left(x_{k}\right)>0$. So $U$ and $V$ are nontrivial solutions of (2.1) and

$$
I_{a, c}(U), I_{a, c}(V) \geq I_{a, c}(W) \text { for any } W \in \mathcal{S}_{a, c} .
$$

Thus, for each $2 R \leq\left|x_{k}\right|$,

$$
\begin{aligned}
I_{a, c}\left(U_{k}\right) & =\frac{1}{N} \int_{\mathbb{R}^{N}}\left|\nabla U_{k}\right|^{2} \geq \frac{1}{N} \int_{B_{R}(0)}\left|\nabla U_{k}\right|^{2}+\frac{1}{N} \int_{B_{R}\left(x_{k}\right)}\left|\nabla U_{k}\right|^{2} \\
& =\frac{1}{N} \int_{B_{R}(0)}\left|\nabla U_{k}\right|^{2}+\frac{1}{N} \int_{B_{R}(0)}\left|\nabla V_{k}\right|^{2} .
\end{aligned}
$$

Taking $R>0$ large enough, we reach a contradiction. Thus, $\lim _{|x| \rightarrow \infty} U(x)=0$ uniformly for $U \in \mathcal{S}_{a, c}$.

Now choose $R$ large and suitable $C$, we see

$$
\begin{aligned}
& U(x) \leq \frac{C}{|R|^{N-2}} \text { on } \partial B_{R}(0) \\
& U(x)<C \text { in } \mathbb{R}^{N} \backslash B_{R}(0) .
\end{aligned}
$$

So

$$
-\Delta U=-\Delta \frac{C}{|x|^{N-2}} \text { in } \mathbb{R}^{N} \backslash B_{R}(0)
$$

and

$$
U(x) \leq \frac{C}{|x|^{N-2}} \text { or } \partial B_{R}(0) .
$$

By the comparison principle, we see that

$$
U(x) \leq \frac{C}{|x|^{N-2}}(\text { if }|x|>R) \quad \text { for any } U \in \mathcal{S}_{a, c} .
$$

At last, we prove that $\mathcal{S}_{a, c}$ is compact in $D^{1,2}\left(\mathbb{R}^{N}\right)$. Let $\left\{U_{k}\right\}_{k=1}^{\infty}$ be a sequence in $\mathcal{S}_{a, c}$. Taking a subsequence if necessary, we can assume that $U_{k}$ converges weakly to some $U$ in $D^{1,2}\left(\mathbb{R}^{N}\right)$. Note that $U$ is solution of $(2.1)$. It is standard to see that as $k \rightarrow \infty$

$$
\int_{|x| \leq \bar{R}} a f\left(U_{k}-c\right) U_{k} \rightarrow \int_{|x| \leq \bar{R}} a f(U-c) U \text { for } \bar{R}>R \text { large. }
$$

Since

$$
\int_{\mathbb{R}^{N}}\left|\nabla U_{k}\right|^{2}-a f\left(U_{k}-c\right) U_{k}=\int_{\mathbb{R}^{N}}|\nabla U|^{2}-a f(U-c) U=0,
$$

it follows from (2.9) that

$$
\lim _{k \rightarrow \infty} \int_{\mathbb{R}^{N}}\left|\nabla U_{k}\right|^{2}=\int_{\mathbb{R}^{N}}|\nabla U|^{2}
$$

This implies that $U_{k} \rightarrow U \in \mathcal{S}_{a, c}$ in $D^{1,2}\left(\mathbb{R}^{N}\right)$ since $U_{k} \rightarrow U \in \mathcal{S}_{a, c}$ weakly in $D^{1,2}\left(\mathbb{R}^{N}\right)$.

As a result, we complete the proof. 


\section{The PROOF OF THE MAIN RESUlts}

In this section, we mainly prove Theorem 1.1 since the proof of Theorem 1.2 is similar. For simplicity, we use $\mathcal{S}_{a}$ to replace $\mathcal{S}_{a, 1}$.

Define

$$
M=\bigcup_{i=1}^{k} M^{i}, O=\bigcup_{i=1}^{k} O^{i}
$$

and for any set $B \subset \Omega, \delta>0, B^{\delta}=\{x \in \Omega$ : $\operatorname{dist}(x, B) \leq \delta\}$. For $u \in H_{\varepsilon}$, let

$$
I_{\varepsilon}(u)=\frac{\varepsilon^{2}}{2} \int_{\Omega}|\nabla u|^{2}-\int_{\Omega} \chi_{O}(x) K(x) f(u-1),
$$

where $\chi_{O}(x)= \begin{cases}1 & x \in O \\ 0 & x \in \Omega \backslash O \text {. }\end{cases}$

We also define

$$
I_{\varepsilon}^{i}(u)=\frac{\varepsilon^{2}}{2} \int_{\Omega}|\nabla u|^{2}-\int_{\Omega} \chi_{O^{i}}(x) K(x) f(u-1) .
$$

It is easy to check that the functionals $I_{\varepsilon}(u)$ and $I_{\varepsilon}^{i}$ are in $C^{1}\left(H_{\varepsilon}\right)$ if we modify $f(t)$ as in $[5]$.

Let

$$
\delta=\frac{1}{10} \min \left\{\operatorname{dist}(M, \Omega \backslash O), \min _{i \neq j} \operatorname{dist}\left(O_{i}, O_{j}\right)\right\} .
$$

We fix $\beta \in(0, \delta)$ and a cutoff $\phi \in C_{0}^{\infty}\left(\mathbb{R}^{N}\right)$ such that $0 \leq \phi \leq 1, \phi(x)=1$ for $|x| \leq \beta$ and $\phi(x)=0$ for $|x|>2 \beta$. For each $y_{i} \in\left(M^{i}\right)^{\beta}$ and $U^{i} \in S_{m_{i}}$, we define

$$
U_{\varepsilon}^{y_{i}, \cdots, y_{k}}=\sum_{i=1}^{k} \phi\left(x-y_{i}\right) U^{i}\left(\frac{x-y_{i}}{\varepsilon}\right) .
$$

We will find a solution near the set

$$
X_{\varepsilon}=\left\{U_{\varepsilon}^{y_{i}, \cdots, y_{k}}(x): y_{i} \in\left(M^{i}\right)^{\beta}, U^{i} \in S_{m_{i}} \text { for each } i=1, \cdots, k\right\} .
$$

For each $i \in\{1, \cdots, k\}$ and $y_{i} \in M^{i}, U^{i} \in \mathcal{S}_{m_{i}}$ fixed, set $W_{\varepsilon}^{i}(x)=\phi\left(x-y_{i}\right) U^{i}\left(\frac{x-y_{i}}{\varepsilon}\right)$ if $y_{i} \in \Omega$. If $y_{i} \in \partial \Omega$, choose $y_{i, \varepsilon} \in \Omega$ such that $\left|y_{i, \varepsilon}-y_{i}\right|=\operatorname{dist}\left\{y_{i, \varepsilon}, \partial \Omega\right\}, \frac{\left|y_{i, \varepsilon}-y_{i}\right|}{\varepsilon|\ln \varepsilon|} \rightarrow$ $c \leq 2 \beta,(c>0)$ as $\varepsilon \rightarrow 0$, and set $W_{\varepsilon}^{i}(x)=\phi\left(\frac{x-y_{i, \varepsilon}}{\varepsilon|\ln \varepsilon|}\right) U^{i}\left(\frac{x-y_{i, \varepsilon}}{\varepsilon}\right)$. Accordingly, define $W_{\varepsilon, t}^{i}(x)=\phi\left(x-y_{i}\right) U^{i}\left(\frac{x-y_{i}}{\varepsilon t}\right)$ or $W_{\varepsilon, t}^{i}(x)=\phi\left(\frac{x-y_{i, \varepsilon}}{\varepsilon|\ln \varepsilon|}\right) U^{i}\left(\frac{x-y_{i, \varepsilon}}{\varepsilon t}\right)$. We see by Proposition 2.5 that $\lim _{t \rightarrow 0}\left\|W_{\varepsilon, t}^{i}\right\|_{\varepsilon}=0$. Moreover, we have

$$
I_{\varepsilon}\left(W_{\varepsilon, t}^{i}\right)=\varepsilon^{N}\left(\frac{t^{N-2}}{2} \int_{\mathbb{R}^{N}}\left|\nabla U^{i}\right|^{2}-t^{N} \int_{\mathbb{R}^{N}} K\left(y_{i}\right) F\left(U^{i}-1\right)\right)+o\left(\varepsilon^{N}\right) .
$$

Thus, there exists $T_{i}>0$ such that $I_{\varepsilon}\left(W_{\varepsilon, t}^{i}\right)=I_{\varepsilon}^{i}\left(W_{\varepsilon, t}^{i}\right)<-2 \varepsilon^{N}$ for $t \geq T_{i}$. 
Let $\gamma_{\varepsilon}^{i}(t)=W_{\varepsilon, t}^{i}(x)$ for $t>0$ and $\gamma_{\varepsilon}^{i}(0)=0$. For $s=\left(s_{1}, \cdots, s_{k}\right) \in T=\left[0, T_{1}\right] \times \cdots \times$ $\left[0, T_{k}\right]$, we define

$$
\gamma_{\varepsilon}(s)=\sum_{i=1}^{k} \gamma_{\varepsilon}^{i}\left(s_{i}\right), D_{\varepsilon}=\max _{s \in T} I_{\varepsilon}\left(\gamma_{\varepsilon}(s)\right) .
$$

For each $c>0$, define $E_{c}=b_{c, 1}$ and $E=\sum_{i=1}^{k} E_{m_{i}}$.

Proposition 3.1. We have

(i) $D_{\varepsilon}-E \varepsilon^{N}=o\left(\varepsilon^{N}\right)$ as $\varepsilon \rightarrow 0$.

(ii) $\limsup _{\varepsilon \rightarrow 0} \max _{s \in \partial T} \varepsilon^{-N} I_{\varepsilon}\left(\gamma_{\varepsilon}(s)\right) \leq \tilde{E}:=\max \left\{E-E_{m_{i}} \mid i=1, \ldots, k\right\}<E$.

(iii) for each $d>0$, there exists $\alpha>0$ such that for sufficiently small $\varepsilon>0, I_{\varepsilon}\left(\gamma_{\varepsilon}(s)\right) \geq$ $D_{\varepsilon}-\alpha \varepsilon^{N}$ implies that $\gamma_{\varepsilon}(s) \in X_{\varepsilon}^{\frac{d}{2} \varepsilon^{\frac{N}{2}}}$, where $X_{\varepsilon}^{\frac{d}{2} \varepsilon^{\frac{N}{2}}}$ is the $\frac{d}{2} \varepsilon^{\frac{N}{2}}$ neighborhood of the set $X_{\varepsilon}$ defined in (3.4).

Proof. Since supp $\gamma_{\varepsilon}(s) \subset M^{2 \beta}$ for each $s \in T$, we see $I_{\varepsilon}\left(\gamma_{\varepsilon}(s)\right)=\sum_{i=1}^{k} I_{\varepsilon}^{i}\left(\gamma_{\varepsilon}^{i}\left(s_{i}\right)\right)$. Now, by Proposition 2.5, we find

$$
\begin{aligned}
\int_{\Omega} \varepsilon^{2}\left|\nabla \gamma_{\varepsilon}\left(s_{i}\right)\right|^{2} & =\varepsilon^{N} s_{i}^{N-2} \int_{\mathbb{R}^{N}} \nabla U^{i 2}+o\left(\varepsilon^{N}\right) \\
\int_{\Omega} \chi_{O^{i}} K(x) f\left(\gamma_{\varepsilon}\left(s_{i}\right)-1\right) & =\varepsilon^{N} s_{i}^{N} \int_{\mathbb{R}^{N}} K\left(y_{i}\right) f\left(U^{i}-1\right)+o\left(\varepsilon^{N}\right) .
\end{aligned}
$$

So, from the Pohozaev identity, we see that

$$
I_{\varepsilon}\left(\gamma_{\varepsilon}^{i}\left(s_{i}\right)\right)=\varepsilon^{N}\left(\frac{s_{i}^{N-2}}{2}-\frac{N-2}{2 N} s_{i}^{N}\right) \int_{\mathbb{R}^{N}} \nabla U^{i}{ }^{2}+o\left(\varepsilon^{N}\right) .
$$

Also $\max _{t \in(0, \infty)}\left(\frac{t^{N-2}}{2}-\frac{N-2}{2 N} t^{N}\right) \int_{\mathbb{R}^{N}}\left|\nabla U^{i}\right|^{2}=E_{m_{i}}$. Hence, (i) and (ii) can be proved easily.

Denote $g(t)=\frac{t^{N-2}}{2}-\frac{N-2}{2 N} t^{N}$, then

$$
g^{\prime}(t) \begin{cases}>0 & t \in(0,1) \\ =0 & t=1 \\ <0 & t>1\end{cases}
$$

and $g^{\prime \prime}(1)=2-N<0$. Hence, (iii) is obvious.

Now define

$$
\Psi_{\varepsilon}^{i}=\left\{\psi \in C\left(\left[0, T_{i}\right], H_{\varepsilon}\right): \psi\left(s_{i}\right)=\gamma_{\varepsilon}^{i}\left(s_{i}\right) \text { for } s_{i}=0 \text { or } s_{i}=T_{i}\right\}
$$

and

$$
C_{\varepsilon}^{i}=\inf _{\psi \in \Psi^{i}} \max _{s_{i} \in\left[0, T_{i}\right]} I_{\varepsilon}^{i}\left(\psi\left(s_{i}\right)\right)
$$

Proposition 3.2. For $i=1, \ldots, k, \lim _{\varepsilon \rightarrow 0} \varepsilon^{-N} C_{\varepsilon}^{i}=E_{m_{i}}$. 
Proof. We prove

$$
\liminf _{\varepsilon \rightarrow 0} \varepsilon^{-N} C_{\varepsilon}^{i} \geq E_{m_{i}} \text { and } \limsup _{\varepsilon \rightarrow 0} \varepsilon^{-N} C_{\varepsilon}^{i} \leq E_{m_{i}} .
$$

Let $\psi\left(s_{i}\right)=\gamma_{\varepsilon}^{i}\left(s_{i}\right), s_{i} \in\left[0, T_{i}\right]$, then we can see

$$
\limsup _{\varepsilon \rightarrow 0} \varepsilon^{-N} C_{\varepsilon}^{i} \leq E_{m_{i}} .
$$

For any $\psi \in \Psi_{\varepsilon}^{i}$, let $\tilde{\psi}\left(s_{i}\right)(x)=\psi\left(s_{i}\right)\left(\varepsilon x+y_{i}\right)$ for $y_{i} \in \Omega, \tilde{\psi}\left(s_{i}\right)(x)=\psi\left(s_{i}\right)\left(\varepsilon x+y_{i, \varepsilon}\right)$ for $y_{i} \in \partial \Omega$, then we see

$$
I_{\varepsilon}^{i}(\psi)=\varepsilon^{N}\left(\frac{1}{2} \int_{\mathbb{R}^{N}} \nabla \tilde{\psi}\left(s_{i}\right)^{2}-\int_{\mathbb{R}^{N}} K\left(y_{i}\right) F\left(\tilde{\psi}\left(s_{i}\right)-1\right)\right)+o\left(\varepsilon^{N}\right)
$$

and $\tilde{\psi}\left(s_{i}\right)$ satisfies $\tilde{\psi}(0)=0$ and $I_{m_{i}, 1}\left(\tilde{\psi}\left(T_{i}\right)\right) \leq-2$.

So

$$
\max _{s_{i} \in\left[0, T_{i}\right]}\left(\frac{1}{2} \int_{\mathbb{R}^{N}} \nabla \tilde{\psi}\left(s_{i}\right)^{2}-\int_{\mathbb{R}^{N}} K\left(y_{i}\right) F\left(\tilde{\psi}\left(s_{i}\right)-1\right)\right) \geq E_{m_{i}} .
$$

Hence $\liminf _{\varepsilon \rightarrow 0} \varepsilon^{-N} C_{\varepsilon}^{i} \geq E_{m_{i}}$.

Proposition 3.3. Let $\left(\varepsilon_{j}\right)$ be such that $\lim _{j \rightarrow \infty} \varepsilon_{j}=0$ and $\left(u_{\varepsilon_{j}}\right) \subset X_{\varepsilon_{j}}^{d \varepsilon_{j}^{\frac{N}{2}}}$ such that

$$
\lim _{j \rightarrow \infty} \varepsilon_{j}^{-N} I_{\varepsilon_{j}}\left(u_{\varepsilon_{j}}\right) \leq E \text { and } \lim \varepsilon_{j}^{-\frac{N}{2}} I_{\varepsilon_{j}}^{\prime}\left(u_{\varepsilon_{j}}\right)=0 \text { in }\left(H_{\varepsilon}\right)^{*} .
$$

Then, for any fixed $d>0$ (sufficiently small), there exists, up to a subsequence, $\left\{z_{j}^{i}\right\} \subset$ $\bar{\Omega}, i=1, \ldots, k, y^{i} \in M^{i}, U^{i} \in \mathcal{S}_{m_{i}}$, such that

$$
\lim _{j \rightarrow \infty} z_{j}^{i}-y^{i}=0, \quad u_{\varepsilon_{j}}-\sum_{i=1}^{k} \phi\left(x-z_{j}^{i}\right) U^{i}\left(\frac{x-z_{j}^{i}}{\varepsilon_{j}}\right)_{\varepsilon}=o\left(\varepsilon_{j}^{\frac{N}{2}}\right) .
$$

Before we prove Proposition 3.3, we give a preliminary lemma.

Lemma 3.4. Let $v \in D^{1,2}\left(\mathbb{R}^{N}\right) \cap C\left(\mathbb{R}^{N}\right)$ satisfy the equation

$$
-\Delta v=\chi_{\left\{x_{1}>0\right\}} f(v-1), \quad x \in \mathbb{R}^{N} .
$$

Then $v \leq 1$ for $x_{1}<0$, so that $v$ actually solves

$$
-\Delta v=f(v-1), \quad x \in \mathbb{R}^{N} .
$$

Proof. Standard regularity arguments yield $v \in C^{1}\left(\mathbb{R}^{N}\right)$ and $\nabla v \rightarrow 0$ as $|x| \rightarrow+\infty$. Using $\frac{\partial v}{\partial x_{1}}$ as a test function, we see

$$
\int_{\mathbb{R}^{N-1}} d x^{\prime} \int_{-\infty}^{+\infty} \frac{\partial}{\partial x_{1}}|\nabla v|^{2} d x_{1}-\int_{\mathbb{R}^{N-1}} F\left(v\left(0, x^{\prime}\right)-1\right) d x^{\prime}=0 .
$$

Noting that the first integral is zero, we obtain $v\left(0, x^{\prime}\right) \leq 1$. Now, to prove that $v \leq 1$ for $x_{1}<0$, we just use $(v-1)_{+} \chi_{\left\{x_{1}<0\right\}} \in D^{1,2}\left(\mathbb{R}^{N}\right)$ as a test function, and obtain that $(v-1)+\chi_{\left\{x_{1}<0\right\}} \equiv 0$. As a result, we complete the proof. 
Proof of Proposition 3.3. For simplicity, we write $\varepsilon$ for $\varepsilon_{j}$. By compactness of $\mathcal{S}_{m_{i}}$ and $M^{\beta}$, there exist $V_{i} \in S_{m_{i}}, z_{j}^{i} \in\left(M^{i}\right)^{\beta}$ and $y^{i} \in\left(M^{i}\right)^{\beta}$ for $i=1, \ldots, k$, such that, passing to a subsequence still denoted by $\left(u_{\varepsilon}\right)$

$$
z_{j}^{i} \rightarrow y^{i}, \quad u_{\varepsilon}-\sum_{i=1}^{k} \phi\left(x-z_{j}^{i}\right) V_{i}\left(\frac{x-z_{j}^{i}}{\varepsilon}\right)_{\varepsilon} \leq 2 d \varepsilon^{\frac{N}{2}}
$$

for $\varepsilon>0$ small enough.

We set

$$
u_{\varepsilon}^{1}=\sum_{i=1}^{k} \phi\left(x-z_{j}^{i}\right) u_{\varepsilon}, \quad u_{\varepsilon}^{2}=u_{\varepsilon}-u_{\varepsilon}^{1}
$$

Now we prove

$$
I_{\varepsilon}\left(u_{\varepsilon}\right) \geq I_{\varepsilon}\left(u_{\varepsilon}^{1}\right)+I_{\varepsilon}\left(u_{\varepsilon}^{2}\right)+o\left(\varepsilon^{N}\right) .
$$

Suppose there exists $y_{\varepsilon} \in A=\bigcup_{i=1}^{k}\left(B\left(y^{i}, 2 \beta\right) \backslash B\left(y^{i}, \beta\right)\right)$ such that $u_{\varepsilon}\left(y_{\varepsilon}\right)>c$ for some $c>0$. Let $v_{\varepsilon}(x)=u_{\varepsilon}\left(\varepsilon x+y_{\varepsilon}\right)$ and $\Omega_{\varepsilon}=\left\{x \in \mathbb{R}^{N}: \varepsilon x+y_{\varepsilon} \in \Omega\right\}$.

Taking a subsequence, we can assume that $y_{\varepsilon} \rightarrow y_{0} \in \bar{A}$. Since (3.10) holds, $\left(v_{\varepsilon}\right)$ is bounded in $D^{1,2}\left(\Omega_{\varepsilon}\right)$, using the blow-up argument (see [18]), we deduce that $v_{\varepsilon}(x) \rightarrow \bar{U}$ weakly in $D^{1,2}\left(\mathbb{R}^{N}\right)$ for some $\bar{U} \in D^{1,2}\left(\Omega_{\infty}\right)$. Moreover, $\bar{U}$ satisfies

$$
-\Delta \bar{U}=K\left(y_{0}\right) f(\bar{U}-1) \text { in } \Omega_{\infty}, \bar{U}(0) \geq c,
$$

where $\Omega_{\infty}=\mathbb{R}_{+}^{N}$ or $\Omega_{\infty}=\mathbb{R}^{N}$. If $\Omega_{\infty}=\mathbb{R}_{+}^{N}$, by Theorem 1.1 in [16], $\bar{U}=0$, which is impossible. So, in (3.12), $\Omega_{\infty}=\mathbb{R}^{N}$. We claim $\max _{x \in \mathbb{R}^{N}} \bar{U}>1$, indeed, if $\bar{U} \leq 1$, then (3.12) implies

$$
-\Delta \bar{U}=0 \text { in } \mathbb{R}^{N}
$$

and hence $\bar{U}=0$, which is a contradiction with $U(0) \geq c$. Hence, by definition, $I_{K\left(y_{0}\right), 1}(\bar{U}) \geq$ $E_{K\left(y_{0}\right)}$. Also, for suitable large $R>0$

$$
\liminf _{\varepsilon \rightarrow 0} \int_{B(y, R)} \varepsilon^{2}\left|\nabla u_{\varepsilon}\right|^{2} \geq \frac{\varepsilon^{N}}{2} \int_{\mathbb{R}^{N}} \nabla \bar{U}^{2} .
$$

It follows from Proposition 2.4 that $E_{K\left(y_{0}\right)} \geq \min \left\{E_{1}, \cdots, E_{k}\right\}$. Thus from Pohozaev identity and (3.13), we get that

$$
\liminf _{\varepsilon \rightarrow 0} \int_{B(y, R)} \varepsilon^{2}\left|\nabla u_{\varepsilon}\right|^{2} \geq \frac{\varepsilon^{N} N}{2} I_{K\left(y_{0}\right), 1}(\bar{U}) \geq \frac{\varepsilon^{N} N}{2} \min \left\{E_{1}, \cdots, E_{k}\right\}>0,
$$

which contradicts (3.10), provided $d>0$ is small enough.

Hence, $\left\|u_{\varepsilon}\right\|_{L^{\infty}(A)} \rightarrow 0$, and

$$
\varepsilon^{-N} \int_{A} K(x) F\left(u_{\varepsilon}-1\right) \rightarrow 0 .
$$

As a consequence,

$$
\lim _{\varepsilon \rightarrow 0} \varepsilon^{-N} \int_{\Omega} \chi_{O} K(x)\left[F\left(u_{\varepsilon}-1\right)-F\left(u_{\varepsilon}^{1}-1\right)-F\left(u_{\varepsilon}^{2}-1\right)\right]=0 .
$$


At this point, write

$$
\begin{aligned}
I_{\varepsilon}\left(u_{\varepsilon}\right)= & I_{\varepsilon}\left(u_{\varepsilon}^{1}\right)+I_{\varepsilon}\left(u_{\varepsilon}^{2}\right)+\int_{\Omega} \varepsilon^{2} \phi(1-\phi)\left|\nabla u_{\varepsilon}\right|^{2} \\
& -\int_{\Omega} \chi_{O} K(x)\left[F\left(u_{\varepsilon}-1\right)-F\left(u_{\varepsilon}^{1}-1\right)-F\left(u_{\varepsilon}^{2}-1\right)\right]+o\left(\varepsilon^{N}\right) .
\end{aligned}
$$

As a result, (3.11) is true.

As the second step, we estimate $I_{\varepsilon}\left(u_{\varepsilon}^{2}\right)$. We will use the blow up technique (see [18]) to prove $I_{\varepsilon}\left(u_{\varepsilon}^{2}\right) \geq 0$.

Since $\left\{u_{\varepsilon}\right\}_{\varepsilon}$ is bounded, we see from (3.10) that $\left\|u_{\varepsilon}^{2}\right\|_{\varepsilon} \leq 4 d \varepsilon^{\frac{N}{2}}$ for small $\varepsilon>0$.

Suppose there exist $y_{\varepsilon} \in \Omega \backslash \bigcup_{i=1}^{k} B\left(y^{i}, 2 \beta\right)$ such that $u_{\varepsilon}^{2}\left(y_{\varepsilon}\right)>c$ for some $c>0$. Let $v_{\varepsilon}(x)=u_{\varepsilon}^{2}\left(\varepsilon x+y_{\varepsilon}\right)$. Taking a subsequence, we can assume that $y_{\varepsilon} \rightarrow y_{0} \in \bar{\Omega} \backslash \bigcup_{i=1}^{k} B\left(y^{i}, 2 \beta\right)$. For the location of $y_{0}$ we have

(1) $y_{0} \in \bar{\Omega} \backslash \bar{O}$

(2) $y_{0} \in \partial O \backslash \partial \Omega, \frac{\left|y-y_{0}\right|}{\varepsilon} \leq \delta$

(3) $y_{0} \in \partial O \backslash \partial \Omega, \frac{\left|y-y_{0}\right|}{\varepsilon} \rightarrow \infty$ as $\varepsilon \rightarrow 0$,

(4) $y_{0} \in \partial \Omega \cap \bar{O}, \frac{\left|y-y_{0}\right|}{\varepsilon} \rightarrow \delta$ as $\varepsilon \rightarrow 0$,

(5) $y_{0} \in \partial \Omega \cap \bar{O}, \frac{\left|y-y_{0}\right|}{\varepsilon} \rightarrow \infty$ as $\varepsilon \rightarrow 0$,

(6) $y_{0} \in O \backslash \bigcup_{i=1}^{k} B\left(y^{i}, 2 \beta\right)$.

Using the blow-up argument, we find that $v_{\varepsilon}(x) \rightarrow U$ weakly in $D^{1,2}\left(\Omega_{\infty}\right)$ for some $\Omega_{\infty} \subset \mathbb{R}^{N}$, and $U$ solves some limit problem.

In case (1), $U$ solves

$$
-\Delta U=0, \quad \Omega_{\infty}=\mathbb{R}^{N} \text { or } \mathbb{R}_{+}^{N} .
$$

Hence, $U=0$ which is impossible since $U(0)>c$.

In case (2), $U$ satisfies

$$
-\Delta U=\chi_{\left\{x_{1} \geq 0\right\}} K\left(y_{0}\right) f(U-1), \Omega_{\infty}=\mathbb{R}^{N} .
$$

By Lemma 3.4, (3.14) is indeed

$$
-\Delta U=K\left(y_{0}\right) f(U-1) \text { in } \mathbb{R}^{N} .
$$

In case (4), $U$ solves

$$
-\Delta U=K\left(y_{0}\right) f(U-1), \text { in } \mathbb{R}_{+}^{N} .
$$

By Theorem 1.1 in [16], $U \equiv 0$, which is impossible.

In cases (3), (5) and (6), $U$ satisfies

$$
-\Delta U=K\left(y_{0}\right) f(U-1) \text { in } \mathbb{R}^{N} .
$$

Summarizing the above analysis, we see $U$ is a solution of

$$
-\Delta U=K\left(y_{0}\right) f(U-1) \text { in } \mathbb{R}^{N} .
$$


Hence, similarly as the proof of (3.11), we see

$$
\left\|u_{\varepsilon}^{2}\right\|_{\varepsilon}^{2} \geq \frac{\varepsilon^{N} N}{2} \min \left\{E_{1}, \cdots, E_{k}\right\}>0
$$

which contradicts the fact $\left\|u_{\varepsilon}^{2}\right\|_{\varepsilon} \leq 4 d \varepsilon^{\frac{N}{2}}$ for $d$ small enough. Hence $\left\|u_{\varepsilon}^{2}\right\|_{L^{\infty}(\Omega)} \rightarrow 0$ and for $\varepsilon$ sufficiently small

$$
I_{\varepsilon}\left(u_{\varepsilon}^{2}\right)=\frac{\varepsilon^{2}}{2} \int_{\Omega} \nabla u_{\varepsilon}^{2}{ }^{2}-\int_{\Omega} \chi_{O} K(x) F\left(u_{\varepsilon}^{2}-1\right)=\frac{\varepsilon^{2}}{2} \int_{\Omega} \nabla u_{\varepsilon}^{2}{ }^{2} \geq 0 .
$$

Now for $i=1, \ldots, k$, we define

$$
u_{\varepsilon}^{1, i}(x)=u_{\varepsilon}^{1}(x) \text { for } x \in O^{i}, u_{\varepsilon}^{1, i}(x)=0 \text { for } x \in \mathbb{R}^{N} \backslash O^{i} .
$$

Set

$$
W_{\varepsilon}^{i}(x)=u_{\varepsilon}^{1, i}(x)\left(\varepsilon x+z_{j}^{i}\right) .
$$

We fix an arbitrary $i \in\{1, \ldots, k\}$. Arguing as before, taking a subsequence, we assume that $W_{\varepsilon}^{i} \rightarrow U^{i}$ weakly in $D^{1,2}\left(\mathbb{R}^{N}\right)$ for some $U^{i} \in D^{1,2}\left(\mathbb{R}^{N}\right)$. Moreover $U^{i}$ solves

$$
-\Delta U^{i}=K\left(y^{i}\right) f\left(U^{i}-1\right), x \in \mathbb{R}^{N} .
$$

From the maximum principle, we see that $U^{i}$ is positive and $\max _{x \in \mathbb{R}^{N}} U^{i}>1$. Now we prove that $W_{\varepsilon}^{i} \rightarrow U^{i}$ strongly in $D^{1,2}\left(\mathbb{R}^{N}\right)$. Suppose that there exists a sequence $\left\{z_{\varepsilon}\right\}_{\varepsilon} \subset B\left(y^{i}, 2 \beta\right)$ satisfying $u_{\varepsilon}^{1, i}\left(z_{\varepsilon}\right)>c$ for some $c>0$ and

$$
\liminf _{\varepsilon \rightarrow \infty} \frac{z_{\varepsilon}-z_{j}^{i}}{\varepsilon}=\infty \text {. }
$$

Proceeding as before, we may assume that $z_{\varepsilon} \rightarrow z_{0} \in \bar{O}$ as $\varepsilon \rightarrow 0$. Then $\tilde{W}_{\varepsilon}^{i}(x)=$ $u_{\varepsilon}^{1, i}\left(\varepsilon x+z_{\varepsilon}\right)$ converges weakly to $\tilde{U}^{i} \in D^{1,2}\left(\mathbb{R}^{N}\right)$ satisfying

$$
-\Delta \tilde{U}^{i}=K\left(z_{0}\right) f\left(\tilde{U}^{i}-1\right), x \in \mathbb{R}^{N},
$$

and as before we get a contradiction. Hence, using $\left(f_{1}\right)\left(f_{2}\right)$ and Lemma 1.1 in [26],

$$
\lim _{\varepsilon \rightarrow 0} \int_{\Omega} \chi_{O^{i}} K\left(\varepsilon x+z_{j}^{i}\right) F\left(W_{\varepsilon}^{i}-1\right)=\int_{\mathbb{R}^{N}} K\left(y^{i}\right) F\left(U^{i}-1\right) .
$$

Now, it follows from the weak convergence of $W_{\varepsilon}^{i}$ to $U^{i}$ in $D^{1,2}\left(\mathbb{R}^{N}\right)$ that

$$
\begin{aligned}
\limsup _{\varepsilon \rightarrow 0} \varepsilon^{-N} I_{\varepsilon}\left(u_{\varepsilon}^{1, i}\right) & \geq \liminf _{\varepsilon \rightarrow 0}\left(\frac{1}{2} \int_{\mathbb{R}^{N}} \nabla W_{\varepsilon}^{i}{ }^{2}-\int_{\mathbb{R}^{N}} K\left(\varepsilon x+y^{i}\right) \chi_{O^{i}} F\left(W_{\varepsilon}^{i}-1\right)\right) \\
& \geq \frac{1}{2} \int_{\mathbb{R}^{N}} \nabla U^{i}-\int_{\mathbb{R}^{N}} K\left(y^{i}\right) F\left(U^{i}-1\right) \\
& \geq E_{m_{i}} .
\end{aligned}
$$

Combining the fact $\lim _{\varepsilon \rightarrow 0} \varepsilon^{-N} I_{\varepsilon}\left(u_{\varepsilon}\right) \leq E$ and (3.11), we derive

$$
\limsup _{\varepsilon \rightarrow 0} \varepsilon^{-N}\left(I_{\varepsilon}\left(u_{\varepsilon}^{2}\right)+\sum_{i=1}^{k} I_{\varepsilon}\left(u_{\varepsilon}^{1, i}\right)\right)=\limsup _{\varepsilon \rightarrow 0} \varepsilon^{-N}\left(I_{\varepsilon}\left(u_{\varepsilon}^{2}\right)+I_{\varepsilon}\left(u_{\varepsilon}^{1}\right)\right)
$$




$$
\begin{aligned}
& \leq \limsup _{\varepsilon \rightarrow 0} \varepsilon^{-N} I_{\varepsilon}\left(u_{\varepsilon}\right) \leq E \\
& =\sum_{i=1}^{k} E_{m_{i}} .
\end{aligned}
$$

Noting $I_{\varepsilon}\left(u_{\varepsilon}^{2}\right) \geq 0$, we see from (3.19) and (3.20)

$$
\lim _{\varepsilon \rightarrow 0} I_{\varepsilon}\left(u_{\varepsilon}^{1, i}\right) \varepsilon^{-N}=E_{m_{i}}, \quad I_{\varepsilon}\left(u_{\varepsilon}^{2}\right)=\frac{1}{2} \quad u_{\varepsilon}^{2}{ }_{\varepsilon}^{2}=o\left(\varepsilon^{N}\right) .
$$

So (3.19) and (3.21) imply

$$
I_{K\left(y^{i}\right), 1}\left(U^{i}\right)=\frac{1}{2} \int_{\mathbb{R}^{N}} \nabla U^{i}{ }^{2}-\int_{\mathbb{R}^{N}} K\left(y^{i}\right) F\left(U^{i}-1\right)=E_{m_{i}} .
$$

It follows from Proposition 2.4 that $y^{i} \in M^{i}$, and as a consequence, $U^{i}(x)=V^{i}\left(x-z_{i}\right)$ with $V^{i} \in \mathcal{S}_{m_{i}}$ and $z_{i} \in \mathbb{R}^{N}$.

Now using (3.18), (3.19), (3.21), we have

$$
\int_{\mathbb{R}^{N}} \nabla U^{i 2} \geq \int_{\mathbb{R}^{N}} \nabla W_{\varepsilon}^{i 2}+o(1) \geq \int_{\mathbb{R}^{N}} \nabla U^{i 2}+o(1)
$$

This proves $\left\|W_{\varepsilon}^{i}-U^{i}\right\|_{D^{1,2\left(\mathbb{R}^{N}\right)}}=o(1)$. In particular, we have

$$
u_{\varepsilon}^{1, i}-U^{i}\left(\frac{x-z_{j}^{i}}{\varepsilon}\right)_{D^{1,2}\left(\mathbb{R}^{N}\right)}^{2}=o\left(\varepsilon^{N}\right) .
$$

Now using the fact $\left\|u_{\varepsilon}^{2}\right\|_{\varepsilon}^{2}=o\left(\varepsilon^{N}\right)$, we complete the proof.

Proposition 3.5. For $d>0$ sufficiently small, there exist constants $b>0$ and $\varepsilon_{0}>0$, such that

$$
\left\|I_{\varepsilon}^{\prime}(u)\right\| \geq b \varepsilon^{\frac{N}{2}} \text { for } u \in I_{\varepsilon}^{D} \cap\left(X_{\varepsilon}^{d \varepsilon^{\frac{N}{2}}} \backslash X_{\varepsilon}^{\frac{d}{2} \varepsilon^{\frac{N}{2}}}\right) \text { and } \varepsilon \in\left(0, \varepsilon_{0}\right),
$$

where $I_{\varepsilon}^{D}=:\left\{u \in H_{\varepsilon}: I_{\varepsilon}(u) \leq D_{\varepsilon}\right\}$.

Proof. To the contrary, we suppose that for small $d>0$, there exist $\left(\varepsilon_{j}\right)$ with $\varepsilon_{j} \rightarrow$ $0(j \rightarrow+\infty)$ and a sequence $\left(u_{\varepsilon_{j}}\right)$ with $u_{\varepsilon_{j}} \in X_{\varepsilon_{j}}^{d \varepsilon_{j}^{\frac{N}{2}}} \backslash X_{\varepsilon_{j}}^{\frac{d}{2} \varepsilon_{j}^{\frac{N}{2}}}$ satisfying

$$
\lim _{j \rightarrow \infty} \varepsilon_{j}^{-N} I_{\varepsilon_{j}}\left(u_{\varepsilon_{j}}\right) \leq E \text { and } \lim _{j \rightarrow 0} \varepsilon_{j}^{-\frac{N}{2}} I_{\varepsilon_{j}}^{\prime}\left(u_{\varepsilon_{j}}\right)=0
$$

By Proposition 3.3, there exists $\left\{z_{j}^{i}\right\} \subset \bar{O}, i=1, \ldots, k, y^{i} \in M^{i}, U^{i} \in S_{m_{i}}$ such that

$$
\begin{gathered}
\lim _{\varepsilon_{j} \rightarrow 0} z_{j}^{i}-y^{i}=0, \\
u_{\varepsilon_{j}}-\sum_{i=1}^{k} \phi\left(x-z_{j}^{i}\right) U^{i}\left(\frac{x-z_{j}^{i}}{\varepsilon_{j}}\right)_{\varepsilon_{j}}^{2}=o\left(\varepsilon_{j}^{N}\right),
\end{gathered}
$$

which is a contradiction with the fact $u_{\varepsilon_{j}} \notin X_{\varepsilon_{j}}^{\frac{d}{2} \varepsilon_{j}^{\frac{N}{2}}}$. So we complete the proof. 
Proposition 3.6. For sufficiently small fixed $\varepsilon>0, I_{\varepsilon}$ has a Palais-Smale sequence in $X_{\varepsilon}^{d \varepsilon^{\frac{N}{2}}} \cap I_{\varepsilon}^{D}$.

Proof. By Proposition 3.1 (iii), there exist $\alpha \in(0, E-\tilde{E})$ such that for sufficiently small $\varepsilon>0$

$$
I_{\varepsilon}\left(\gamma_{\varepsilon}(s)\right) \geq D_{\varepsilon}-\alpha \varepsilon^{N} \Rightarrow \gamma_{\varepsilon}(s) \in X_{\varepsilon}^{\frac{d}{2} \varepsilon^{\frac{N}{2}}} .
$$

If Proposition 3.6 does not hold for small $\varepsilon>0$, there exists $a(\varepsilon)>0$ such that $\left\|I_{\varepsilon}^{\prime}(u)\right\| \geq a(\varepsilon)$ on $X_{\varepsilon}^{d \varepsilon^{\frac{N}{2}}} \cap I_{\varepsilon}^{D}$. Also, we know from Proposition 3.5 that

$$
\left\|I_{\varepsilon}^{\prime}(u)\right\|>b \varepsilon^{\frac{N}{2}}, \forall u \in I_{\varepsilon}^{D} \cap\left(X_{\varepsilon}^{d \varepsilon^{\frac{N}{2}}} \backslash X_{\varepsilon}^{\underline{d} \varepsilon^{\frac{N}{2}}}\right),
$$

where $b$ is independent of $\varepsilon$.

Now there exists a pseudo-gradient vector field $\mathcal{P}_{\varepsilon}$ on a neighborhood $Z_{\varepsilon}$ of $X_{\varepsilon}^{d \varepsilon^{\frac{N}{2}}} \cap I_{\varepsilon}^{D}$ for $I_{\varepsilon}$ (see [37]). Let $\eta_{\varepsilon}$ be a Lipschitz continuous function on $H_{\varepsilon}$ such that $0 \leq \eta_{\varepsilon} \leq 1$, $\eta_{\varepsilon} \equiv 1$ on $X_{\varepsilon}^{d \varepsilon^{\frac{N}{2}}} \cap I_{\varepsilon}^{D}$ and $\eta_{\varepsilon} \equiv 0$ on $H_{\varepsilon} \backslash Z_{\varepsilon}$.

Let $\xi_{\varepsilon}$ be also a Lipschitz continuous on $\mathbb{R}$ such that $0 \leq \xi_{\varepsilon} \leq 1, \xi_{\varepsilon}(t) \equiv 0$ if $\left|t-D_{\varepsilon}\right| \geq$ $\alpha \varepsilon^{N}$, and $\xi_{\varepsilon}(t) \equiv 1$ if $\left|t-D_{\varepsilon}\right| \leq \frac{\alpha}{2} \varepsilon^{N}$. Then, there exists a global solution $\Phi_{\varepsilon}: H_{\varepsilon} \times \mathbb{R} \rightarrow H_{\varepsilon}$ of the initial value problem

$$
\begin{aligned}
\frac{\partial}{\partial t} \Phi_{\varepsilon}(u, t) & =-\eta_{\varepsilon}\left(\Phi_{\varepsilon}(u, t)\right) \xi_{\varepsilon}\left(I_{\varepsilon}\left(\Phi_{\varepsilon}(u, t)\right)\right) \\
\Phi_{\varepsilon}(u, 0) & =u .
\end{aligned}
$$

Now, we can choose $\tau_{\varepsilon}>0$ large, such that for some $\mu \in(0, \alpha)$,

$$
I_{\varepsilon}\left(\Phi_{\varepsilon}\left(\gamma_{\varepsilon}(s), \tau_{\varepsilon}\right)\right) \leq D_{\varepsilon}-\mu \varepsilon^{N}, s \in T .
$$

Let $\gamma(s)=\Phi_{\varepsilon}\left(\gamma_{\varepsilon}(s), \tau_{\varepsilon}\right)$. We also have

$$
\gamma(s)=\gamma_{\varepsilon}(s) \text { for } \gamma_{\varepsilon}(s) \in I_{\varepsilon}^{D-\alpha \varepsilon^{N}} .
$$

Let $\phi \in C_{0}^{\infty}\left(\mathbb{R}^{N}\right)$ satisfy that $\phi(x)=1$ for $x \in O^{\delta}, \phi(x)=0$ for $x \notin O^{2 \delta}, \phi(x) \in[0,1]$, and $|\nabla \phi| \leq \frac{2}{\delta}$. Define $\gamma^{1}(s)=\phi \gamma(s)$ and $\gamma^{2}(s)=(1-\phi) \gamma(s)$. By the definition of $I_{\varepsilon}$, we see

$$
\begin{aligned}
I_{\varepsilon}(\gamma(s)) & =I_{\varepsilon}\left(\gamma^{1}(s)\right)+\frac{\varepsilon^{2}}{2} \int_{\Omega} \nabla \gamma^{2}(s)^{2}+\int_{\Omega} \varepsilon^{2} \phi(1-\phi)|\nabla \gamma(s)|^{2} \\
& \geq I_{\varepsilon}\left(\gamma^{1}(s)\right) .
\end{aligned}
$$

For $i=1, \cdots, k$, set $\gamma^{1, i}(s)(x)=\gamma^{1}(s)(x)$ for $x \in\left(O^{i}\right)^{\delta}, \gamma^{1 . i}(s)(x)=0$ for $x \notin\left(O^{i}\right)^{\delta}$, we see

$$
I_{\varepsilon}\left(\gamma^{1}(s)\right)=\sum_{i=1}^{k} I_{\varepsilon}\left(\gamma^{1, i}(s)\right)=\sum_{i=1}^{k} I_{\varepsilon}^{i}\left(\gamma^{1, i}(s)\right)
$$


By Proposition $3.1 I_{\varepsilon}\left(\gamma_{\varepsilon}(s)\right) \leq \varepsilon^{N} \tilde{E}+o\left(\varepsilon^{N}\right)<D_{\varepsilon}-\alpha \varepsilon^{N}, s \in \partial T$, so $\gamma_{\varepsilon}(s) \in I_{\varepsilon}^{D-\alpha \varepsilon^{N}} \forall s \in$ $\partial T$. Using (3.23) we see $\gamma(s)=\gamma_{\varepsilon}(s)$ on $\partial T$ and hence $\gamma^{1, i}(s) \in \Psi_{\varepsilon}^{i}$ for $i=1, \ldots, k$. Therefore, by Proposition 3.2, (3.24) and (3.25), we see

$$
\max _{s \in T} I_{\varepsilon}(\gamma(s)) \geq \sum_{i=1}^{k} E_{m_{i}} \varepsilon^{N}+o\left(\varepsilon^{N}\right)=D_{\varepsilon}+o\left(\varepsilon^{N}\right),
$$

which contradicts (3.22) if $\varepsilon$ is sufficiently small. So we complete the proof.

Proof of Theorem 1.1. By Proposition 3.6, for a fixed small $\varepsilon$, we obtain a Palais-Smale sequence $\left\{u_{n}\right\}_{n=1}^{\infty}$ in $X_{\varepsilon}^{d \varepsilon^{\frac{N}{2}}} \cap I_{\varepsilon}^{D}$, so it follows from Proposition 3.3 that $\left\{u_{n}\right\}_{n=1}^{\infty}$ is bounded in $H_{\varepsilon}$. Suppose that $u_{n} \rightarrow u_{\varepsilon}$ weakly in $H_{\varepsilon}$, then $u_{\varepsilon}$ is a critical point of $I_{\varepsilon}$. Now we prove $u_{\varepsilon} \in X_{\varepsilon}^{d \varepsilon^{\frac{N}{2}}} \cap I_{\varepsilon}^{D}$. Write $u_{n}=v_{n}+w_{n}, v_{n} \in X_{\varepsilon},\left\|w_{n}\right\|_{\varepsilon} \leq d \varepsilon^{\frac{N}{2}}$. Since $X_{\varepsilon}$ is compact, there exists $v_{\varepsilon} \in X_{\varepsilon}$ such that $v_{n} \rightarrow v_{\varepsilon}$ in $X_{\varepsilon}$ up to a subsequence, as $n \rightarrow \infty$. Now suppose $w_{n} \rightarrow w_{\varepsilon}$ weakly in $H_{\varepsilon}$, then $u_{\varepsilon}=w_{\varepsilon}+v_{\varepsilon}$ and

$$
\left\|u_{\varepsilon}-v_{\varepsilon}\right\|_{\varepsilon}=\left\|w_{\varepsilon}\right\|_{\varepsilon} \leq \liminf _{n \rightarrow \infty}\left\|w_{n}\right\|_{\varepsilon} \leq d \varepsilon^{\frac{N}{2}} .
$$

Hence $u_{\varepsilon} \in X_{\varepsilon}^{d \varepsilon^{\frac{N}{2}}}$.

To show $u_{\varepsilon} \in I_{\varepsilon}^{D}$, it suffices to prove

$$
\liminf _{n \rightarrow \infty} I_{\varepsilon}\left(u_{n}\right) \geq I_{\varepsilon}\left(u_{\varepsilon}\right) .
$$

Let $q_{n}=u_{n}-u_{\varepsilon}$. Since $u_{n}$ and $u_{\varepsilon}$ are in $X_{\varepsilon}^{d \varepsilon^{\frac{N}{2}}}$, we see by triangular inequality that

$$
\left\|q_{n}\right\|_{\varepsilon} \leq 2 d \varepsilon^{\frac{N}{2}}+o_{n}(1) \text {. }
$$

It follows from $u_{n} \rightarrow u_{\varepsilon}$ weakly in $H_{\varepsilon}$ that

$$
\left\|q_{n}\right\|_{\varepsilon}^{2}=\left\|u_{n}\right\|_{\varepsilon}^{2}-\left\|u_{\varepsilon}\right\|_{\varepsilon}^{2}+o_{n}(1) .
$$

On the other hand, it follows from $\left(f_{1}\right)$ that

$$
\int_{\Omega} F\left(u_{n}-c\right)=\int_{\Omega} F\left(u_{\varepsilon}-c\right)+o_{n}(1),
$$

which implies (3.26) immediately.

Now $u_{\varepsilon}$ is a critical point of $I_{\varepsilon}(u)$, and $u_{\varepsilon}$ solves

$$
-\varepsilon^{2} \Delta u_{\varepsilon}=\chi_{O} K(x) f\left(u_{\varepsilon}-1\right) \text { in } \Omega .
$$

By Proposition 3.3, we see

$$
\int_{\Omega \backslash M^{\delta}} \varepsilon^{2}\left|\nabla u_{\varepsilon}\right|^{2} \leq o\left(\varepsilon^{N}\right) .
$$

Performing Moser iteration scheme, we deduce

$$
\left\|u_{\varepsilon}\right\|_{L^{\infty}\left(\Omega \backslash M^{\delta}\right)}=o(1) \text {, as } \varepsilon \rightarrow 0^{+} .
$$


So, for $\varepsilon$ sufficiently small, $u_{\varepsilon}$ is indeed a solution of

$$
-\varepsilon^{2} \Delta u=K(x) f(u-1) \text { in } \Omega .
$$

Let $x_{\varepsilon}^{i}$ be a maximum point of $u_{\varepsilon}$ in $O^{i}$. Set $v_{\varepsilon}=u_{\varepsilon}\left(x_{\varepsilon}^{i}+\varepsilon x\right)$, then from the proof of Proposition 3.3, we see, after passing a subsequence, $x_{\varepsilon}^{i} \rightarrow x^{i} \in M^{i}$ and that $v_{\varepsilon}$ converges in the $C^{2}$ sense over compact sets to a positive solution $U^{i} \in D^{1,2}\left(\mathbb{R}^{N}\right)$ of equation (1.4). Moreover, using the arguments to prove Theorem 0.1 in [14], we deduce that $x_{\varepsilon}^{i}$ is the unique local maximum point of $u_{\varepsilon}$ in $O^{i}$. Choose $R_{i}$ such that

$$
v_{\varepsilon}(x) \leq c \leq 2 U^{i}(x) \text {, if }|x|=R_{i},
$$

which implies that

$$
u_{\varepsilon}(x) \leq c \leq 2 U^{i}\left(\frac{x-x_{\varepsilon}^{i}}{\varepsilon}\right), \forall x \in \partial B_{\varepsilon R_{i}}\left(x_{\varepsilon}^{i}\right)
$$

and

$$
u_{\varepsilon}(x) \leq c \leq 2 \sum_{i=1}^{k} U^{i}\left(\frac{x-x_{\varepsilon}^{i}}{\varepsilon}\right) \leq C \varepsilon^{N-2} \max _{i=1, \ldots, k} x-x_{\varepsilon}^{i{ }^{2-N}}, \forall x \in \bigcup_{i=1}^{k} \partial B_{\varepsilon R_{i}}\left(x_{\varepsilon}^{i}\right),
$$

for some $C>0$ independent of $U^{i}$ and $\varepsilon$.

On the other hand, from the proof of Proposition 3.3, we see

$$
u_{\varepsilon}(x) \leq c, \quad \forall x \in \Omega \backslash \bigcup_{i=1}^{k} B_{\varepsilon R_{i}}\left(x_{\varepsilon}^{i}\right),
$$

which implies that

$$
-\varepsilon^{2} \Delta u_{\varepsilon}(x)=0, \quad \forall x \in \Omega \backslash \bigcup_{i=1}^{k} B_{\varepsilon R_{i}}\left(x_{\varepsilon}^{i}\right) .
$$

Using the comparison principle, we see that

$$
u_{\varepsilon}(x) \leq C \varepsilon^{N-2} \max _{i=1, \ldots, k} x-x_{\varepsilon}^{i^{2-N}}, \forall x \in \Omega \backslash \bigcup_{i=1}^{k} B_{\varepsilon R_{i}}\left(x_{\varepsilon}^{i}\right) .
$$

As a consequence,

$$
u_{\varepsilon}(x) \leq C \varepsilon^{N-2} \max _{i=1, \ldots, k} x-x_{\varepsilon}^{i}{ }^{2-N}, \forall x \in \Omega \backslash \bigcup_{i=1}^{k} B_{\delta}\left(x_{\varepsilon}^{i}\right) .
$$

At last, noting (3.9) and the fact

$$
U^{i}\left(\frac{x-z_{j}^{i}}{\varepsilon}\right)-\phi\left(x-z_{j}^{i}\right) U^{i}\left(\frac{x-z_{j}^{i}}{\varepsilon}\right)_{\varepsilon}^{2}=o\left(\varepsilon^{N}\right),
$$

we can derive part (ii) of Theorem 1.1 directly. 
Proof of Theorem 1.2. Since we have Propositions 2.4 and 2.5, we can prove Theorem 1.2 with the same arguments as Theorem 1.1.

Acknowledgements: S. Peng would like to thank the Department of Mathematics at Wright State University for its hospitality during his visit. This work was partially supported by the funds from NSFC( NO.11125101) and Program for Changjiang Scholars and Innovative Research Team in University(NO.IRT13066).

\section{REFERENCES}

[1] A. Ambrosetti, G. Mancini, On some free boundary problems, Recent contributions to nonlinear partial differential equations, Res. Notes in Math., 50, Pitman, Boston, Mass.-London, 1981, pp. $24-36$.

[2] A. Ambrosetti, M. Struwe, Existence of steady vortex rings in an ideal fluid, Arch. Rational Mech. Anal., 108(1989), 97-109.

[3] T. V. Badiani, Existence of steady symmetric vortex pairs on a planar domain with an obstacle, Math. Proc. Cambridge Philos. Soc., 123(1998), 365-384.

[4] C. Bandle, M. Marcus, Remarks on plasma problems with nonmonotone nonlinearities, Applicable Anal., 17(1983), 63-78.

[5] H. Berestycki, P. L. Lions, Nonlinear scalar field equations I, Arch. Rational Mech. Anal., 82(1983), 313-346.

[6] M. S. Berger, L. E. Fraenkel, Nonlinear desingularization in certain free-boundary problems, Comm. Math. Phys., 77(1980), 149-172.

[7] J. Byeon, L. Jeanjean, Standing waves for nonlinear Schrödinger equations with a general nonlinearity, Arch. Rational Mech. Anal., 185(2007), 185-200.

[8] L. Caffarelli, A. Friedman, Asymptotic estimates for the plasma problem, Duke Math. J., 47(1980), $705-742$.

[9] D. Cao, S. Peng, S. Yan, Multiplicity of solutions for the plasma problem in two dimensions, Adv. Math., 225(2010), 2741-2785.

[10] V. Coti Zelati, I. Ekeland, E. Sere, A variational approach to homoclinic orbits in Hamiltonian systems, Math. Ann., 288(1990), 133-160.

[11] V. Coti Zelati, P. H. Rabinowitz, Homoclinic type solutions for a semilinear elliptic PDE on $\mathbb{R}^{n}$, Comm. Pure Appl. Math., 45(1992), 1217-1269.

[12] F. Flucher, J. Wei, Asymptotic shape and location of small cores in elliptic free-boundary problems, Math. Z., 228(1998), 638-703.

[13] E. N. Dancer, S. Yan, The Lazer-McKenna conjecture and a free boundary problem in two dimensions, J. Lond. Math. Soc., 78(2008), 639-662.

[14] M. del Pino, P. Felmer, Local mountain passes for semilinear elliptic problems in umbounded domains, Calc. Var. Partial Differential Equations, 4(1996), 121-137.

[15] M. del Pino, P. Felmer, Multi-peak bound states for nonlinear Schrödinger equations, Ann. Inst. H. Poincaré Anal. Non Linéaire, 15(1998), 127-149.

[16] M. J. Esteban, P. L. Lions, Existence and nonexistence results for semilinear elliptic problems in unbounded domains, Proc. Roy. Soc. Edinburgh Sect. A, 93(1982), 1-14.

[17] L. E. Fraenkel, M. S. Berger, A global theory of steady vortex rings in an ideal fluid, Acta Math., 132(1974), 13-51.

[18] B. Gidas, J. Spruck, A priori bounds for positive solutions of nonlinear elliptic equations, Comm. Partial Differential Equations, 6(1981), 883-901. 
[19] D. Gilbarg, N. S. Trudinger, Elliptic partial differential of second order; second edition, Grundlehren 224, Springer, Berlin, Heidelberg, New York and Tokyo, 1983.

[20] C. Gui, Existence of multi-bump solutions for nonlinear Schrödinger equations via variational method, Comm. Partial Differential Equations, 21(1996), 787-820.

[21] L. Jeanjean, K. Tanaka, A remark on least energy solutions in $\mathbb{R}^{N}$, Proc. Amer. Math. Soc., 131(2003), 2399-2408.

[22] D. Kinderlehrer, L. Nirenberg, J. Spruck, Regularity in elliptic free boundary problems, J. Anal. Math., 34(1978), 86-119.

[23] D. Kinderlehrer, J. Spruck, The shape and smoothness of stable plasma configurations, Ann. Scuola Norm. Sup. Pisa Cl. Sci., 5(1978), 131-148.

[24] G. Li, S. Yan, J.Yang, An elliptic problem related to planar vortex pairs, SIAM J. Math. Anal., 36(2005), 1444-1460.

[25] Y. Li, S. Peng, Multiple solutions for an elliptic problem related to vortex pairs, J. Differential Equations, 250(2011), 3448-3472.

[26] P. L. Lions, The concentration-compactness principle in the calculus of variations, the locally compact case, part II, Ann. Inst. Henri Poincaré,1(1984), 223-283.

[27] W. M. Ni, On the existenct of global vortex rigs, J. Anal. Math., 37(1980), 208-247.

[28] J. Norbury, Steady planar vortex pairs in an ideal fluid, Comm. Pure Appl. Math., 28(1975), 679-700.

[29] M. Ramos, H. Tavares, W. Zou, A Bahri-Lions theorem revisited, Adv. Math., 222(2009), 2173-2195.

[30] E. Sere, Existence of infinitely many homoclinic orbits in Hamiltonian systems, Math. Z., 209(1992), $27-42$.

[31] M. Shibata, Asymptotic shape of a solution for the plasma problem in higher dimensional spaces, Commun. Pure Appl. Anal., 2(2003), 259-275.

[32] M. Shibata, Asymptotic shape of a least energy solution to an elliptic free-boundary problem with non-autonomous nonlinearity, Asymptot. Anal., 31(2002), 1-42.

[33] R. Temam, A nonlinear eigenvalue problem: The shape at equilibrium of a confined plasma, Arch. Rational Mech. Anal.,60(1975), 51-73.

[34] R. Temam, Remarks on a free boundary value problem arising in plasma physics, Comm. Partial Differential Equations, 2(1977), 263-585.

[35] B. Turkington, On steady vortex flow in two dimensions I, II, Comm. Partial Differential Equations, 8(1983), 999-1071.

[36] J. Wei, Multiple condensations for a nonlinear elliptic equation with sub-critical growth and critical behaviour, Proc. Edinb. Math. Soc., 44(2001), 631-660.

[37] M. Willem, Minimax theorems, Progress in nonlinear differential equations and their applications, 24. Birkhäuser Boston, Inc., Boston,1996.

[38] J. Yang, Existence and asymptotic behavior in planar vortex theory, Math. Models Methods Appl. Sci., 1(1991), 461-475.

Department of Mathematics and Statistics, Wright State University, Dayton, OH45435, U.S.A

E-mail address: yi.li@wright.edu

School of Mathematics and Hubei Key laboratory of Mathematical Physics, Central China Normal University, Wuhan, 430079, P. R. China

E-mail address: sjpeng@mail.ccnu.edu.cn 